\title{
COMPACTNESS PROPERTIES FOR PERTURBED SEMIGROUPS AND APPLICATION TO TRANSPORT EQUATION
}

\author{
KHALID LATRACH \\ (Received 16 March 1998; revised 17 January 2000)
}

Communicated by P. G. Dodds

\begin{abstract}
Using the comparison results for positive compact operators by Aliprantis and Burkinshow, MokhtarKharroubi investigated compactness properties of positive semigroups on Banach lattices. The aim of this paper is to study these properties in general Banach spaces (without positivity). Our results generalize a part of those obtained by Mokhtar-Kharroubi to general Banach spaces context. More specifically, we derive conditions which ensure the compactness of the remainder term $R_{n}(t)$ for some integer $n$. The improvement here is that it can applied directly to the neutron transport equation for a wide class of collision operators.
\end{abstract}

2000 Mathematics subject classification: primary 47A10, 47A55, 47G20.

\section{Introduction}

Let $X$ be a Banach space and let $\mathscr{L}(X)$ be the set of all bounded linear operators in $X$. Let $T$ be the infinitesimal generator of a $C^{0}$-semigroup $\{U(t), t \geq 0\}$ acting on $X$. We denote by $\omega$ the type of this semigroup. It is well known (see, for instance, $[6,10])$ that if $K \in \mathscr{L}(X)$, then $T+K$ generates a strongly continuous semigroup $\{V(t), t \geq 0\}$ given by the Dyson-Phillips expansion

$$
V(t)=\sum_{j=0}^{\infty} U_{j}(t),
$$

where

$$
U_{0}(t)=U(t), \quad U_{j}(t)=\int_{0}^{t} U(s) K U_{j-1}(t-s) d s \quad(j \geq 1) .
$$

(C) 2000 Australian Mathematical Society $0263-6115 / 2000 \$ A 2.00+0.00$ 
The remainder term of order $n$ is given by

$$
R_{n}(t)=\sum_{j=n}^{\infty} U_{j}(t)=\int_{\substack{s_{1}+\cdots+s_{n} \leq t, s_{i} \geq 0}} U\left(s_{1}\right) K \cdots U\left(s_{n}\right) K V\left(t-\sum_{i=1}^{n} s_{i}\right) d s_{1} \cdots d s_{n} .
$$

For a linear operator $T$ acting on $X$, the spectrum of $T$ is denoted by $\sigma(T)$. The resolvent set of $T$ is the complement of $\sigma(T)$ in the complex plane, $\rho(T)=\mathbb{C} \backslash \sigma(T)$. For any $\lambda$ in $\rho(T)$, the resolvent operator $(\lambda-T)^{-1}$ is denoted by $R(\lambda, T)$.

In his classical work [12], Vidav proved that under the following condition

$$
\left\{\begin{array}{l}
\text { there exists } n \geq 1 \text { such that }\left[(\lambda-T)^{-1} K\right]^{n} \text { is compact for all } \lambda \\
\text { satisfying } \operatorname{Re} \lambda>\omega
\end{array}\right.
$$

the part of the spectrum of $T+K$ lying in the half plane $\operatorname{Re} \lambda>\omega$ consists of, at most, isolated eigenvalues with finite algebraic multiplicity. Unfortunately, this condition does not guarantee that the part of the spectrum of the perturbed semigroup $V(t)$ outside the spectral disc of $U(t)$ consists of, at most, isolated eigenvalues. This is due to the fact that, in general, we have $e^{t \sigma(T+K)} \subseteq \sigma(V(t))$. More precisely, there can exist a point $\alpha \neq 0$ in the continuous spectrum of $V(t)$ which does not belong to the closure of the set $e^{t \sigma(T+K)}$. So, it is necessary to attack the problem directly by studying the spectrum of the operators $\{V(i), t>0\}$. Motivated by problems in transport theory, a perturbation technique for studying the spectrum of $V(t)$ was initiated by Vidav [13]. His analysis was clarified and refined later by Voigt [14] who showed that if

$$
\left\{\begin{array}{l}
\text { there exist } m, n \in \mathbb{N} \text { such that }\left(R_{n}(t) B\right)^{m} \text { is compact for large } t \text { and } \\
\text { for all } B \in \mathscr{L}(X),
\end{array}\right.
$$

then $\sigma(V(t)) \cap\left\{\alpha \in \mathbb{C}:|\alpha|>e^{\omega t}\right\}$ consists of, at most, isolated eigenvalues with finite algebraic multiplicity. In particular, for any $\nu>\omega, \sigma(T+K) \cap\{\lambda \in \mathbb{C}: \operatorname{Re} \lambda>v\}$ consists of, at most, finitely many eigenvalues $\left\{\lambda_{1}, \ldots, \lambda_{n}\right\}$. If $P_{i}$ and $D_{i}$ denote, respectively, the spectral projection and the nilpotent operator associated with $\lambda_{i}$, $1 \leq i \leq n$, then

$$
V(t)=(I-P) V(t)+\sum_{i=1}^{n} e^{\lambda_{i} t} e^{D_{i} t} P_{i}
$$

with $\|(I-P) V(t)\|=o\left(e^{\left(\lambda^{\prime}-\varepsilon\right) t}\right)$ as $t \rightarrow+\infty$, where $P=\sum_{i=1}^{n} P_{i}, \lambda^{\prime}=\min \left\{\operatorname{Re} \lambda_{i}\right.$, $1 \leq i \leq p\}$ and $\varepsilon>0$ small enough.

Within the framework of positive semigroups, Mokhtar-Kharroubi [8] has shown the existence of many connections between the assumptions ( $\mathrm{H} 1)$ and $(\mathrm{H} 2)$. In particular, the spectral analysis of the perturbed semigroup $V(t)$ is possible with assumptions such as (H1). 
The aim of this paper is to generalize and to extend a part of the results obtained in [8] to general Banach space context. Indeed, we prove that if there exists $n \in \mathbb{N}$, $n \neq 0$ such that if

$$
\left\{\begin{array}{l}
K \prod_{i=1}^{n}\left(R(\lambda, T) U\left(t_{i}\right) K\right) \text { is compact for all } n \text {-tuples }\left(t_{1}, \ldots, t_{n}\right), t_{i}>0 \\
\left(t_{1}, \ldots, t_{n}\right) \rightarrow K \prod_{i=1}^{n}\left(U\left(t_{i}\right) K\right) \text { is continuous in the uniform topology }
\end{array}\right.
$$

then the remainder $R_{n+1}(t)$ of order $n+1$ in the Dyson-Phillips expansion $V(t)=$ $\sum_{j=0}^{\infty} U_{j}(t)$ is compact. This shows that the spectral analysis of the perturbed semigroup $V(t)$, is also possible on general Banach spaces (without positivity). Our methods of proofs are different from those used in [8] and do not rely on positivity arguments.

In Section 2 we discuss, under the assumption (H3), the compactness of the remainders $R_{n}(t)$ of order $n$ in the Dyson-Phillips expansion. The main results of Section 2 are Theorem 2.1 and Theorem 2.2. We close Section 2 by giving a spectral analysis of perturbed semigroups. Finally, applications of these results to the neutron transport equation are the topic of Section 3.

\section{Compactness results}

Let $X$ be a Banach space and let $T$ be the infinitesimal generator of a $C^{0}$-semigroup $(U(t), t \geq 0)$ of type $\omega$ acting on $X$. Let $K \in \mathscr{L}(X)$. Following the perturbation theory (see [6,10]), $A=T+K$ generates a $C^{0}$-semigroup which we denote by $(V(t), t \geq 0)$.

LEMMA 2.1. The following two statements are equivalent :

(i) The operator $U(t) K$ is compact on $X$ for every $t>0$.

(ii) The map $(0,+\infty) \ni t \rightarrow U(t) K$ is continuous in the uniform topology and $R(\lambda, T) K$ is compact on $X$ for some (every) $\lambda$ in $\rho(T)$.

ProOF. Let $M$ be such that $\|T(t)\| \leq M e^{\omega t}$. Assume $U(t) K$ is compact for every $t>0$. Since $K$ is bounded and located on the right of $U(t)$, arguing as in the proof of $[10$, Theorem 3.2 , page 48$]$ we get the continuity of the map $(0, \infty) \rightarrow U(t) K$ in the uniform topology.

We now prove the second part of the assertion. Indeed, it follows from boundedness of $K$ that

$$
R(\lambda, T) K=\int_{0}^{\infty} e^{-\lambda t} U(t) K d t, \quad \text { for } \operatorname{Re} \lambda>\omega
$$

and the integral exists in the uniform operator topology. 
Let $\delta>0, \operatorname{Re} \lambda>\omega$ and

$$
W(\lambda, \delta)=\int_{\delta}^{\infty} e^{-\lambda t} U(t) K d t
$$

Since $U(t) K$ is compact for every $t>0$, the use of [16, Corollary 2.3] implies the compactness of $W(\lambda, \delta)$. Whence, the estimate

$$
\|R(\lambda, T) K-W(\lambda, \delta)\| \leq\left\|\int_{0}^{\delta} e^{-\lambda t} U(t) K d t\right\| \leq \delta M\|K\| \rightarrow 0 \quad \text { as } \delta \rightarrow 0
$$

shows the compactness of $R(\lambda, T) K$. Now the resolvent identity gives the compactness of $R(\lambda, T) K$ for every $\lambda \in \rho(T)$.

Conversely, let $t>0$, since $U(t) K$ is bounded we may write

$$
\lambda R(\lambda, T) U(t) K=\int_{0}^{\infty} \lambda e^{-\lambda s} U(t+s) K d s \quad \text { for every } \lambda>\omega .
$$

Further, the use of the relation $\int_{0}^{\infty} \lambda e^{-\lambda s} d s=1$ leads to

$$
\lambda R(\lambda, T) U(t) K-U(t) K=\int_{0}^{\infty} \lambda e^{-\lambda \hat{q}}\{U(t+s) K-U(t) K] d s .
$$

Let $\lambda>\omega$. Then for every $\delta>0$ we have

$$
\begin{aligned}
\|\lambda R(\lambda, T) U(t) K-U(t) K\| \\
\quad \leq \int_{0}^{\infty} \lambda e^{-\lambda s}\|U(t+s) K-U(t) K\| d s \\
\quad=\int_{0}^{\delta} \lambda e^{-\lambda s}\|U(t+s) K-U(t) K\| d s+\int_{\delta}^{\infty} \lambda e^{-\lambda s}\|U(t+s) K-U(t) K\| d s \\
\quad \leq \sup _{0 \leq s \leq \delta}\|U(t+s) K-U(t) K\|+\lambda M\|K\| e^{\omega t}\left[(\lambda-\omega)^{-1} e^{-(\lambda-\omega) \delta}+\lambda^{-1} e^{-\lambda \delta}\right] .
\end{aligned}
$$

Therefore, we get

$$
\lim _{\lambda \rightarrow \infty}\|\lambda R(\lambda, T) U(t) K-U(t) K\| \leq \sup _{0 \leq s \leq \delta}\|U(t+s) K-U(t) K\|
$$

for every $\delta>0$. Since $\delta>0$ is arbitrary, we have

$$
\lim _{\lambda \rightarrow \infty}\|\lambda R(\lambda, T) U(t) K-U(t) K\|=0 .
$$

Finally, the compactness of $U(t) K(t>0)$ follows from the commutativity of the operators $R(\lambda, T)$ and $U(t)$ and $(2.1)$. 
We are now prepared to establish the following result.

THEOREM 2.1. Suppose that the map $U(\cdot) K:(0, \infty) \rightarrow \mathscr{L}(X), t \rightarrow U(t) K$ is continuous in the uniform topology and that, for some $\lambda$ in $\rho(T), R(\lambda, T) K$ is compact. Then

$$
V(t)-U(t)=\int_{0}^{t} U(t-s) K V(s) d s
$$

is compact on $X$.

PROOF. The result is immediate if $t=0$. Suppose now $t>0$. Clearly, Lemma 2.1 implies the compactness of $U(t) K$. Accordingly, the operator $U(s) K V(t-s)$ is compact too for every $s \in(0, t]$. Therefore, using Duhamel formula [6, equation (2.3), page 497] and applying [16, Corollary 2.3] one concludes the compactness of $V(t)-$ $U(t)$ for every $t>0$.

REMARK 2.1. A theorem of the type of Theorem 2.1 was first proved by Vidav ([13, Theorem 1]). In his special situation $K$ is compact and $X$ is reflexive. Later, assuming the compactness of $K$, Vidav's analysis was improved and extended by Voigt ([14, Theorem 2.4]) to the case of general Banach spaces. Note, however, that Voigt's hypothesis is stronger than those of Theorem 2.1. In fact, if $K$ is compact, then $U(t) K$ is also compact and consequently the assertion of Theorem 2.1 follows via Lemma 2.1.

REMARK 2.2. It should be noted that the result of Theorem 2.1 holds provided that the map $(0,+\infty) \ni t \rightarrow K U(t)$ is continuous in the uniform topology and, for some $\lambda$ in $\rho(T), K R(\lambda, T)$ is compact on $X$. Indeed, under these hypotheses, for all $t>0$, the operator $K U(t)$ is compact. To see this, let $t>0$ and let $\lambda>\omega$. Using the boundedness of $K U(t)$ we obtain

$$
\lambda K U(t) R(\lambda, T)-K U(t)=\int_{0}^{\infty} \lambda e^{-\lambda s}[K U(t+s)-K U(t)] d s .
$$

Similar calculations as in the second part of the proof of Lemma 2.1 lead to

$$
\lim _{\lambda \rightarrow \infty}\|\lambda K U(t) R(\lambda, T)-K U(t)\|=0 .
$$

Using the commutativity of the operators $U(t)$ and $R(\lambda, T)$ and the fact that $K R(\lambda, T)$ is compact, we infer from the last equation the compactness of $K U(t)(t>0)$. 
On the other hand, the operator $T$ may be viewed as a bounded perturbation of $T+K$ by $-K$. Hence according to Duhamel formula we have

$$
U(t)=V(t)+\int_{0}^{t} V(t-s)(-K) U(s) d s .
$$

From the compactness of $K U(\tau),(\tau>0)$, we infer that $V(t-s)(-K) U(s)$ is compact for each $s \in(0, t]$. Applying [16, Corollary 2.3] we obtain the compactness of $R_{1}(t)=V(t)-U(t)$.

COROLLARY 2.1. Assume that the hypotheses of Theorem 2.1 are satisfied. Then the map $(0, \infty) \ni t \rightarrow R_{1}(t) K$ is continuous in the uniform topology.

PROOF. By virtue of the hypotheses, it suffices to show the continuity in the uniform topology of the map $(0,+\infty) \ni t \rightarrow V(t) K$. To do this, we first observe that Lemma 2.1 and Theorem 2.1 imply the compactness of $U(t) K$ and $R(t) K$ for all $t>0$. Therefore, $V(t) K$ is compact for all $t>0$. Proceeding as in the first part of the proof of Lemma 2.1 we obtain the continuity in the uniform topology of the map $(0,+\infty) \ni t \rightarrow V(t) K$ which completes the proof.

REMARK 2.3. We have seen above that $U(t) K$ î̀ continuous in the uniform topology if its values are compact operators for each $t>0$ (Lemma 2.1). It seems that in case $n \geq 2$, the compactness of $U\left(t_{1}\right) K \cdots U\left(t_{n}\right) K$ for each $n$-tuple $\left(t_{1}, \ldots, t_{n}\right) \in(0, \infty)^{n}$ does not suffice. Nevertheless, for $n \geq 2$, it is not difficult to check that, if

$$
\left\{\begin{array}{l}
K \prod_{i=2}^{n}\left(U\left(t_{i}\right) K\right) \text { is compact for all }(n-1) \text {-tuples }\left(t_{2}, \ldots, t_{n}\right), t_{i}>0 \\
\left(t_{2}, \ldots, t_{n}\right) \rightarrow K \prod_{i=2}^{n}\left(U\left(t_{i}\right) K\right) \text { is continuous in the uniform topology, }
\end{array}\right.
$$

then the map $\left(t_{1}, \ldots, t_{n}\right) \rightarrow K \prod_{i=1}^{n}\left(U\left(t_{i}\right) K\right)$ is continuous in the uniform topology and its values are compact operators on $X$.

Indeed, let $\left(t_{1}^{\prime}, \ldots, t_{n}^{\prime}\right)$ and $\left(t_{1}, \ldots, t_{n}\right)$ be two elements of $(0, \infty)^{n}$. Then

$$
\begin{aligned}
& K U\left(t_{1}^{\prime}\right) K \cdots K U\left(t_{n}^{\prime}\right) K-K U\left(t_{1}\right) K \cdots K U\left(t_{n}\right) K \\
& =K U\left(t_{1}^{\prime}\right)\left[K U\left(t_{2}^{\prime}\right) \cdots K U\left(t_{n}^{\prime}\right)-K U\left(t_{2}\right) \cdots K U\left(t_{n}\right)\right] \\
& \quad+K\left[U\left(t_{1}^{\prime}\right)-U\left(t_{1}\right)\right] K U\left(t_{2}\right) K \cdots U\left(t_{n}\right) K .
\end{aligned}
$$

The first term of the right member tends to zero in norm as $\left(t_{2}^{\prime}, \ldots, t_{n}^{\prime}\right) \rightarrow\left(t_{2}, \ldots, t_{n}\right)$. Since $U(t)$ is bounded and $(U(t), t>0)$ is continuous in the strong operator topology, the use of the compactness of $K U\left(t_{2}\right) K \cdots K U\left(t_{n}\right) K(t>i)$ and [6, Lemma 3.7, page 151] implies that the second term of the right member also tends to zero in norm as $t_{1}^{\prime}$ tends to $t_{1}$ which completes the proof of the statement.

Now, we come to the main result of this section. 
THEOREM 2.2. Let $n \geq 1$ be a fixed integer and suppose that, for all $\lambda>\omega$, the following assumption is satisfied

$$
\left\{\begin{array}{l}
K \prod_{i=1}^{n}\left(R(\lambda, T) U\left(t_{i}\right) K\right) \text { is compact for all } n \text {-tuples }\left(t_{1}, \ldots, t_{n}\right), t_{i}>0 \\
\left(t_{1}, \ldots, t_{n}\right) \rightarrow K \prod_{i=1}^{n}\left(U\left(t_{i}\right) K\right) \text { is continuous in the uniform topology. }
\end{array}\right.
$$

Then the remainder term $R_{n+1}(t)$ of the Dyson-Phillips expansion is compact on $X$ for all $t \geq 0$.

REMARK 2.4. Note that Theorem 2.2 generalizes Theorem 6 in [8] to general Banach spaces. Indeed, the latter theorem was proved in the Banach lattice context and its proof is essentially based on comparison arguments for positive operators (see [1]).

The proof of Theorem 2.2 uses the following lemma.

LEMMA 2.2. Under the assumptions of Theorem 2.2 the operator $K \prod_{i=1}^{n}\left(U\left(t_{i}\right) K\right)$ is compact on $X$ for all $n$-tuples $\left(t_{1}, \ldots, t_{n}\right), t_{i}>0$ with $i=1, \ldots, n$.

For the sake of simplicity, we consider only the case $n=2$. The general case can be treated similarly.

PROOF. Since $\lambda \in \rho(T)$, we can write

$$
\begin{aligned}
\lambda^{2} K R & (\lambda, T) U\left(t_{1}\right) K R(\lambda, T) U\left(t_{2}\right) K-K U\left(t_{1}\right) K U\left(t_{2}\right) K \\
= & \lambda^{2} \int_{0}^{\infty} K e^{-\lambda t} U\left(t+t_{1}\right) d t \int_{0}^{\infty} K e^{-\lambda s} K U\left(s+t_{2}\right) K d s-K U\left(t_{1}\right) K U\left(t_{2}\right) K \\
= & \lambda^{2} \int_{0}^{\infty} \int_{0}^{\infty} e^{-\lambda(t+s)} K U\left(t+t_{1}\right) K U\left(s+t_{2}\right) K d t d s \\
& -\lambda^{2} \int_{0}^{\infty} \int_{0}^{\infty} e^{-\lambda(t+s)} K U\left(t_{1}\right) K U\left(t_{2}\right) K d t d s \quad\left(\text { because } \int_{0}^{\infty} \lambda e^{-\lambda t} d t=1\right) \\
= & \lambda^{2} \int_{0}^{\infty} \int_{0}^{\infty} e^{-\lambda(t+s)}\left[K U\left(t+t_{1}\right) K U\left(s+t_{2}\right) K-K U\left(t_{1}\right) K U\left(t_{2}\right) K\right] d t d s .
\end{aligned}
$$

Let $\delta>0$. Writing

$$
J\left(t, s, t_{1}, t_{2}\right)=\lambda^{2} e^{-\lambda(t+s)}\left[K U\left(t+t_{1}\right) K U\left(s+t_{2}\right) K-K U\left(t_{1}\right) K U\left(t_{2}\right) K\right]
$$

we have

$$
\begin{aligned}
\int_{0}^{\infty} \int_{0}^{\infty} J\left(t, s, t_{1}, t_{2}\right) d t d s \\
=\int_{0}^{\delta} \int_{0}^{\delta} J\left(t, s, t_{1}, t_{2}\right) d t d s+\int_{0}^{\delta} d t \int_{\delta}^{\infty} J\left(t, s, t_{1}, t_{2}\right) d t d s \\
\quad+\int_{\delta}^{\infty} d t \int_{0}^{\delta} J\left(t, s, t_{1}, t_{2}\right) d t d s+\int_{\delta}^{\infty} \int_{\delta}^{\infty} J\left(t, s, t_{1}, t_{2}\right) d t d s
\end{aligned}
$$


Setting

$$
\begin{array}{ll}
I_{1}=\int_{0}^{\delta} \int_{0}^{\delta} J\left(t, s, t_{1}, t_{2}\right) d t d s, & I_{3}=\int_{\delta}^{\infty} \int_{0}^{\delta} J\left(t, s, t_{1}, t_{2}\right) d t d s, \\
I_{2}=\int_{0}^{\delta} \int_{\delta}^{\infty} J\left(t, s, t_{1}, t_{2}\right) d t d s, & I_{4}=\int_{\delta}^{\infty} \int_{\delta}^{\infty} J\left(t, s, t_{1}, t_{2}\right) d t d s
\end{array}
$$

we get

$$
\int_{0}^{\infty} \int_{0}^{\infty} J\left(t, s, t_{1}, t_{2}\right) d t d s=I_{1}+I_{2}+I_{3}+I_{4}
$$

Using the estimate

$$
\begin{aligned}
\left\|I_{1}\right\| & \leq \int_{0}^{\delta} \int_{0}^{\delta} \lambda^{2} e^{-\lambda(t+s)}\left\|K U\left(t+t_{1}\right) K U\left(s+t_{2}\right) K-K U\left(t_{1}\right) K U\left(t_{2}\right) K\right\| d s d t \\
& \leq \sup _{0 \leq t, s \leq \delta}\left\|K U\left(t+t_{1}\right) K U\left(s+t_{2}\right) K-K U\left(t_{1}\right) K U\left(t_{2}\right) K\right\|\left(\int_{0}^{\delta} \lambda e^{-\lambda t} d t\right)^{2}
\end{aligned}
$$

and $\int_{0}^{\delta} \lambda e^{-\lambda t} d t \leq 1$, we obtain

$$
\left\|I_{1}\right\| \leq \sup _{0 \leq t, s \leq \delta}\left\|K U\left(t+t_{1}\right) K U\left(s+t_{2}\right) K-K U\left(t_{1}\right) K U\left(t_{2}\right) K\right\| .
$$

Next,

$$
\begin{aligned}
\left\|I_{2}\right\| & \leq \int_{0}^{\delta} \int_{\delta}^{\infty} \lambda^{2} e^{-\lambda(t+s)}\left\|K U\left(t+t_{1}\right) K U\left(s+t_{2}\right) K-K U\left(t_{1}\right) K U\left(t_{2}\right) K\right\| d s d t \\
& \leq \int_{\delta}^{\infty} \lambda e^{-\lambda s} \sup _{0 \leq t \leq \delta}\left\|K U\left(t+t_{1}\right) K U\left(s+t_{2}\right) K-K U\left(t_{1}\right) K U\left(t_{2}\right) K\right\| d s \int_{0}^{\delta} \lambda e^{-\lambda t} d t .
\end{aligned}
$$

Obviously, the estimate

$$
\begin{aligned}
\sup _{0 \leq s \leq \delta} & \left\|K U\left(t+t_{1}\right) K U\left(s+t_{2}\right) K-K U\left(t_{1}\right) K U\left(t_{2}\right) K\right\| \\
& \leq\|K\|^{3} M^{2} e^{\omega\left(t_{1}+t_{2}+t+\delta\right)}+\|K\|^{3} M^{2} e^{\omega\left(t_{1}+t_{2}\right)}=\|K\|^{3} M^{2} e^{\omega\left(t_{1}+t_{2}\right)}\left[1+e^{\omega(t+\delta)}\right]
\end{aligned}
$$

and $\int_{\delta}^{\infty} e^{-\lambda t} e^{\omega(t+\delta)} d t=e^{\omega \delta}\left(e^{-(\lambda-\omega) \delta}\right) /(\lambda-\omega)$ lead to

$$
\left\|I_{2}\right\| \leq \int_{\delta}^{\infty} \lambda e^{-\lambda t}\|K\|^{3} M^{2} e^{\omega\left(t_{1}+t_{2}+t+\delta\right)} d t+\int_{\delta}^{\infty} \lambda e^{-\lambda t}\|K\|^{3} M^{2} e^{\omega\left(t_{1}+t_{2}\right)} d t .
$$

Since $\int_{\delta}^{\infty} \lambda e^{-\lambda t} d t=e^{-\lambda \delta}$, we obtain

$$
\left\|I_{2}\right\| \leq\|K\|^{3} M^{2} e^{\omega\left(t_{1}+t_{2}\right)}\left[e^{-\lambda \delta}+\frac{e^{-\delta(\lambda-2 \omega)}}{\lambda-\omega}\right] .
$$


Note that $I_{3}$ is similar to $I_{2}$, then it satisfies the estimate (2.2).

Consider now $I_{4}$. It is easy to see that

$$
\begin{aligned}
\left\|I_{4}\right\| \leq & \int_{\delta}^{\infty} \int_{\delta}^{\infty} \lambda^{2} e^{-\lambda(t+s)}\|K\|^{3} M^{2} e^{\omega\left(t_{1}+t_{2}+t+s\right)} d t d s \\
& +\int_{\delta}^{\infty} \int_{\delta}^{\infty} \lambda^{2} e^{-\lambda(t+s)}\|K\|^{3} M^{2} e^{\omega\left(t_{1}+t_{2}\right)} d t d s
\end{aligned}
$$

Hence, from the relation

$$
\begin{aligned}
& \int_{\delta}^{\infty} \int_{\delta}^{\infty} \lambda^{2} e^{-\lambda(t+s)}\|K\|^{3} M^{2} e^{\omega\left(t_{1}+t_{2}+t+s\right)} d t d s \\
& \quad=\|K\|^{3} M^{2} e^{\omega\left(t_{1}+t_{2}\right)}\left\{\int_{\delta}^{\infty} \int_{\delta}^{\infty} \lambda e^{-(\lambda-\omega) s} d s\right\}^{2} \\
& =\|K\|^{3} M^{2} e^{\omega\left(t_{1}+t_{2}\right)}\left(\frac{\lambda}{\lambda-\omega}\right)^{2} e^{-2(\lambda-\omega) \delta}
\end{aligned}
$$

and

$$
\begin{aligned}
& \int_{\delta}^{\infty} \int_{\delta}^{\infty} \lambda^{2} e^{-\lambda(t+s)}\|K\|^{3} M^{2} e^{\omega\left(t_{1}+t_{2}\right)} d t d s \\
& \quad=\|K\|^{3} M^{2} e^{\omega\left(t_{1}+t_{2}\right)}\left\{\int_{\delta}^{\infty} \int_{\delta}^{\infty} \lambda e^{-\lambda s} d s\right\}^{2}=\|K\|^{3} M^{2} e^{\omega\left(t_{1}+t_{2}\right)} e^{-2 \lambda \delta}
\end{aligned}
$$

it follows that

$$
\left\|I_{4}\right\| \leq\|K\|^{3} M^{2} e^{\omega\left(t_{1}+\ell_{2}\right)}\left[e^{-2 \lambda \delta}+\frac{\lambda^{2} e^{-2(\lambda-\omega) \delta}}{(\lambda-\omega)^{2}}\right] .
$$

Since $\delta>0$ is arbitrary we have $\left\|I_{1}\right\|=0$. Moreover, the estimates (2.2) and (2.3) imply

$$
\lim _{\lambda \rightarrow \infty}\left\|I_{i}\right\|=0 \text { for } i=2,3,4
$$

Consequently, we have

$$
\lim _{\lambda \rightarrow \infty}\left\|\lambda^{2} K R(\lambda, T) U\left(t_{1}\right) K R(\lambda, T) U\left(t_{2}\right) K-K U\left(t_{1}\right) K U\left(t_{2}\right) K\right\|=0 .
$$

Now, the use of the compactness of $K R(\lambda, T) U\left(t_{1}\right) K R(\lambda, T) U\left(t_{2}\right) K$ together with (2.4) gives the compactness of $K U\left(t_{1}\right) K U\left(t_{2}\right) K$. This completes the proof.

ProOF OF THEOREM 2.2. For $t=0$, the result is obvious. Let $t>0$. By Lemma 2.2 we have the compactness of $K U\left(t_{1}\right) K U\left(t_{2}\right) \cdots K U\left(t_{n}\right) K$ on $X$. Hence the integrand of $R_{n+1}(t), t>0$ is compact on $X$. Now, the use of [16, Corollary 2.3] ends the proof. 
Let $T$ be a closed, densely defined linear operator on $X$. We recall that $\lambda \in \mathbb{C}$ is an eigenvalue of finite algebraic multiplicity of $T$ if $\lambda$ is an isolated point of $\sigma(T)$ and is a pole of the resolvent of $T$ with degenerate associated spectral projection $P$ (see [6, III, 6.5]), where $\sigma(T)$ denotes the spectrum of $T$.

Let $B \in \mathscr{L}(X)$. The essential spectral radius of $B$ is defined by $r_{e}(B):=\sup \{|\lambda|, \lambda \in \sigma(B), \lambda$ but is not an eigenvalue of finite algebraic multiplicity $\}$. Let $\{U(t), t \geq 0\}$ be a strongly continuous semigroup on $X$. Then

$$
r(U(t))=e^{t \omega} \quad \text { for every } t>0,
$$

where $r(\cdot)$ denotes the spectral radius and $\omega$ is the type of $\{U(t), t \geq 0\}$, that is,

$$
\omega=\lim _{t \rightarrow \infty} \frac{1}{t} \log \|U(t)\|=\inf _{t>0} \frac{1}{t} \log \|U(t)\| .
$$

Further, there exists $\omega_{e} \in[-\infty, \omega]$ such that

$$
r_{e}(U(t))=e^{t \omega_{e}} \quad(t>0)
$$

(see [14, Lemma 2.1]). The real number $\omega_{e}$ is called the essential type of $(U(t), t \geq 0)$. Let us first recall a known result on the essential type of perturbed $C^{0}$-semigroups on Banach spaces.

PROPOSITION 2.1 ([14, Theorem 2.2]). With the notations introduced above, suppose that there exist two integers $m$ and $n$ and $t_{0} \geq 0$ such that $\left(B R_{n}(t)\right)^{m}$ is compact for $t \geq t_{0}$ and for all $B \in \mathscr{L}(X)$. Then $r_{e}(V(t)) \leq e^{t \omega}$, where $\{V(t), t \geq 0\}$ is the $C^{0}$-semigroup generated by $T+K$.

COROLLARY 2.2. Assume that the hypotheses of Theorem 2.2 are satisfied. Then $\sigma(V(t)) \cap\left\{\mu \in \mathbb{C}:|\mu|>e^{t \omega}\right\}$ consists of (at most) isolated eigenvalues with finite algebraic multiplicities.

REMARK 2.5. The interest of Corollary 2.2 lies in the fact that it implies $\omega_{e} \leq \omega$. Such a property is useful for the description of the large time behaviour of $V(t)$. In fact, the part of the spectrum of $V(t)$ outside the spectral disc of $U(t)$ can consist only of eigenvalues of finite algebraic multiplicities. Assuming the existence of such eigenvalues, the semigroup $V(t)$ can be decomposed into two parts, the first containing the time development of finitely many eigenmodes, the second being of faster decay.

PROOF OF COROLlaRY 2.2. Clearly the hypotheses of Theorem 2.2 imply the compactness of $R_{n+1}(t)$ for all $t \geq 0$ and $n \geq 1$. Therefore, for all $B \in \mathscr{L}(X)$ and $m \geq 1$, $\left(B R_{n+1}(t)\right)^{m}$ is compact on $X$. Now the use of Proposition 2.1 gives the desired assertion.

REMARK 2.6. Corollary 2.2 extends [8, Theorem 5] to general Banach spaces. 


\section{Application to neutron transport equation}

The purpose of this section is to apply the previous results to the neutron transport equation. Indeed, consider the integro-differential operator

$$
\left\{\begin{aligned}
A \psi(x, \xi) & =-\xi \frac{\partial \psi}{\partial x}(x, \xi)-\sigma(\xi) \psi(x, \xi)+\int_{V} \kappa\left(x, \xi, \xi^{\prime}\right) \psi\left(x, \xi^{\prime}\right) d \xi^{\prime} \\
& =T \psi+K \psi
\end{aligned}\right.
$$

where $T$ is the streaming operator and $K$ denotes the integral part of $A$ (the collision operator), $(x, \xi) \in D \times V$, where the configuration space $D$ is an open bounded subset of $\mathbb{R}^{N}(N \geq 1)$, while the velocity space $V$ is an arbitrary open subset of $\mathbb{R}^{N}$. The unbounded operator $A$ is studied in the Banach spaces $L_{p}(D \times V), 1 \leq p<\infty$.

It's domain is given by

$$
D(A)=\left\{\psi \in L_{p}(D \times V), \xi \frac{\partial \psi}{\partial x} \in L_{p}(D \times V), \psi_{\left.\right|_{\Gamma_{-}}}=0\right\},
$$

where $\Gamma_{-}=\{(x, \xi) \in \partial D \times V, \xi$ is ingoing at $x \in \partial D\}$. For the trace results we refer, for instance, to $[3,4]$.

The usual assumptions are

$$
\sigma(\cdot) \in L^{\infty}(V) \text {-and } K \in \mathscr{L}\left(L_{p}(D \times V)\right) .
$$

It is well known (see, for instance, [3, 9]) that the streaming operator $T=A-K$ generates the following explicit $C^{0}$-semigroup

$$
U(t) \psi(x, \xi)= \begin{cases}e^{-t \sigma(\xi)} \psi(x-t \xi, \xi) & \text { if } t<s(x, \xi) \\ 0 & \text { otherwise, }\end{cases}
$$

where $s(x, \xi)=\inf \{s>0, x-s \xi \notin D\}$. Since $K$ is bounded in $L_{p}(D \times V), T+K$ generates a $C^{0}$-semigroup $\{V(t), t>0\}$ (see $\left.[6,10]\right)$.

REMARK 3.1. In the neutron transport theory the compactness of $R_{2}(t)(t>0)$ on $L_{p}(D \times V)(1<p<\infty)$ was considered in the 1980 s by many authors under different assumptions and conditions on the scattering kernels (see, for instance, [5, 11, 15]). Recently, the same result was proved in [9] for a wide and useful class of collision operators referred to as regular operators (see [9, Definition 2.1]). In what follows we give another proof of this result based solely on the results obtained in Section 2.

Note that the operator $K$ is local in $x$ so it can be viewed as a mapping

$$
K(\cdot): x \in D \rightarrow K(x) \in \mathscr{L}\left(L_{p}(V)\right) .
$$


We assume that $K(\cdot)$ is strongly measurable,

$$
x \in D \rightarrow K(x) f \in L_{p}(V) \text { is measurable for any } f \in L_{p}(V)
$$

and bounded

$$
\text { ess } \sup _{x \in D}\|K(x)\|_{\mathscr{L}_{\left(L_{p}(V)\right)}<\infty .}
$$

It follows easily that $K$ defines a bounded operator on the space $L_{p}(D \times V)$ according to the rule

$$
\varphi \in L_{p}(D \times V) \rightarrow K(x) \varphi(x) \in L_{p}(D \times V)
$$

(we identify $L_{p}(D \times V)$ and $\left.L_{p}\left(D ; L_{p}(V)\right)\right)$ and

$$
\|K(x)\|_{\mathscr{L}\left(L_{p}(D \times V)\right)} \leq \mathrm{ess} \sup _{x \in D}\|K(x)\|_{\mathscr{L}\left(L_{p}(V)\right)} .
$$

Our final assumption is

$$
K(x) \in \mathscr{K}\left(L_{p}(V)\right) \quad \text { almost everywhere, }
$$

where $\mathscr{K}\left(L_{p}(V)\right)$ denotes the set of all compact operators on $L_{p}(V)$.

DEFINITION 3.1 ([9, Definition 2.1]). A collision operator

$$
K(\cdot): x \in D \rightarrow K(x) \in \mathscr{L}\left(L_{p}(V)\right) .
$$

is said to be regular if $K(x) \in \mathscr{K}\left(L_{p}(V)\right)$ almost everywhere on $D$ and

$$
K(\cdot): x \in D \rightarrow K(x) \in \mathscr{L}\left(L_{p}(V)\right)
$$

is Bochner measurable.

In the sequel, we denote by $\mathscr{R}\left(L_{p}(D \times V)\right)$ the set of all regular collision operators on $L_{p}(D \times V)$. The interest of this class of operators lies in the following lemma.

LEMMA 3.1 ([9, Lemma 2.3]). A regular collision operator $K$ can be approximated, in the uniform topology, by a sequence $\left\{K_{n}\right\}$ of collision operators with kernels of the form

$$
\sum_{i \in I} f_{i}(x) g_{i}(\xi) h_{i}\left(\xi^{\prime}\right)
$$

where $f_{i} \in L^{\infty}(D), g_{i} \in L^{p}(V)$ and $h_{i} \in L^{p^{\prime}}(V)\left(1 / p+1 / p^{\prime}=1\right)$ (I is finite). 
As it is suggested by the hypotheses of Theorem 2.2 we define the set $\mathscr{P}\left(L_{p}(D \times V)\right)$ $(1 \leq p<\infty)$ by

$$
\begin{aligned}
\mathscr{P}\left(L_{p}(D \times V)\right)= & \left\{K \in \mathscr{L}\left(L_{p}(D \times V)\right) \text { such that there exists } n \in N^{*}\right. \text { satisfying } \\
& K \prod_{i=1}^{n}\left(R(\lambda, T) U\left(t_{i}\right) K\right)\left(t_{i}>0\right) \text { is compact on } L_{p}(D \times V) \\
& \text { and the map }\left(t_{1}, \ldots, t_{n}\right) \rightarrow K \prod_{i=1}^{n}\left(U\left(t_{i}\right) K\right) \text { is continuous } \\
& \text { in the uniform topology }\} .
\end{aligned}
$$

First we prove the following result.

THEOREM 3.1. Let $p \in[1, \infty)$ and $K \in \mathscr{P}\left(L_{p}(D \times V)\right)$. Then there exists an integer $n$ such that $R_{n+1}(t)$ is compact for all $t \geq 0$ on $L_{p}(D \times V)$.

PROOF. The result follows immediately from Theorem 2.2 .

The next corollary shows the relationship between the sets $\mathscr{P}\left(L_{p}(D \times V)\right)$ and $\mathscr{R}\left(L_{p}(D \times V)\right)$.

COROLlaRY 3.1. Let $p \in(1, \infty)$. With the notations above, we have

$$
\mathscr{R}\left(L_{p}(D \times V)\right) \subseteq \mathscr{P}\left(L_{p}(D \times V)\right) \text {. }
$$

PROOF. Let $K \in \mathscr{R}\left(L_{p}(D \times V)\right)$ and let $\lambda \in \rho(T)$. Using [9, Lemma 2.1] we deduce the compactness of $K(\lambda-T)^{-1}$ on $L_{p}(D \times V)$ and consequently the operator $K(\lambda-T)^{-1} U(t) K$ is compact on $L_{p}(D \times V)$ for all $t>0$. On the other hand, [7, Theorem 1] implies the continuity in the uniform topology of the map $(0, \infty) \ni t \rightarrow K U(t) K$. Hence we conclude that $K \in \mathscr{P}\left(L_{p}(D \times V)\right)$ which completes the proof.

It follows from Corollary 3.1 and Theorem 4.1 that if $K \in \mathscr{R}\left(L_{p}(D \times V)\right)$, then there exists an integer $n \geq 2$ such that $R_{n}(t), t \geq 0$ is compact on $L_{p}(D \times V)$. In fact, we have the following more precise result.

COROLlaRY 3.2. Let $p \in(1, \infty)$ and let $K \in \mathscr{R}\left(L_{p}(D \times V)\right)$. Then $R_{2}(t)$ is compact on $L_{p}(D \times V)$ for all $t \geq 0$.

PROOF. Let $K \in \mathscr{R}\left(L_{p}(D \times V)\right)$ and let $\lambda \in \rho(T)$. Clearly (see the proof of Corollary 4.1) we have the compactness of $K(\lambda-T)^{-1} U(t) K(t>0)$ on $L_{p}(D \times V)$ and the continuity in the uniform topology of the map $(0, \infty) \ni t \rightarrow K U(t) K$. Thus the result follows from Theorem 2.2.

REMARK 3.2. The Corollary 4.2 is the first part of Theorem 3.1 in [9]. 
Let us now consider the case $p=1$. To avoid some technical difficulties, we assume that $D$ is convex.

THEOREM 3.2. For $p=1$ we have

$$
\mathscr{R}\left(L_{1}(D \times V)\right) \subseteq \mathscr{P}\left(L_{1}(D \times V)\right) .
$$

PROOF. Let $K \in \mathscr{R}\left(L_{1}(D \times V)\right)$. We claim that $K R(\lambda, T) U(t) K$ is compact on $L_{1}(D \times V)$, for every $\lambda \in \rho(T)$ and $t>0$. Indeed, by Lemma 4.1 there exists a sequence of finite rank operators which converges, in the operator norm, to $K$. Then it suffices to establish the result for a finite rank operator, that is, $\kappa\left(x, \xi, \xi^{\prime}\right)=\sum_{i=1}^{n} \kappa_{i}\left(x, \xi, \xi^{\prime}\right), \kappa_{i}\left(x, \xi, \xi^{\prime}\right)=f_{i}(x) g_{i}(\xi) h_{i}\left(\xi^{\prime}\right)$, where $n \in N^{*}, f_{i}(\cdot) \epsilon$ $L^{\infty}(D), g_{i}(\cdot) \in L^{1}(V)$ and $h_{i}(\cdot) \in L^{\infty}(V)$. Since the compactness is stable by summation, we can restrict ourselves to an operator of kernel $\kappa_{i}\left(x, \xi, \xi^{\prime}\right)$ (which we denote again by $K)$. Thus, $K R(\lambda, T) U(t) K$ is an integral operator on $L_{1}(D \times V)$ whose kernel is

$$
\begin{aligned}
\int_{t}^{\infty} e^{-\left(\lambda+\sigma\left(\left(x-x^{\prime}\right) / s\right)\right) s} f_{i}(x) g_{i}(\xi) h_{i}\left(\frac{x-x^{\prime}}{s}\right) f_{i}\left(x^{\prime}\right) g_{i}\left(\frac{x-x^{\prime}}{s}\right) h_{i}\left(\xi^{\prime}\right) \frac{d s}{s^{N}} \\
=f_{i}(x) g_{i}(\xi) N_{\lambda}^{t}\left(x, x^{\prime}\right) f_{i}\left(x^{\prime}\right) h_{i}\left(\xi^{\prime}\right), \ldots
\end{aligned}
$$

where

$$
N_{\lambda}^{t}\left(x, x^{\prime}\right)=\int_{t}^{\infty} e^{-\left(\lambda+\sigma\left(\left(x-x^{\prime}\right) / s\right)\right) s} J_{i}\left(\frac{x-x^{\prime}}{s}\right) \frac{d s}{s^{N}}
$$

with $J_{i}(\cdot)=g_{i}(\cdot) h_{i}(\cdot) \in L^{1}\left(R^{N}\right)\left(J_{i}(\cdot)\right.$ is extended by zero outside $\left.V\right)$. It follows that $K R(\lambda, T) U(t) K=A_{4} A_{2} A_{3} A_{2} A_{1}$, where

$$
\begin{aligned}
& \left\{\begin{array} { l l } 
{ A _ { 1 } : } & { L ^ { 1 } ( D \times V ) \rightarrow L ^ { 1 } ( D ) } \\
{ } & { \psi ( x , \xi ) \rightarrow \int _ { V } h _ { i } ( \xi ) \psi ( x , \xi ) d \xi , }
\end{array} \left\{\begin{array}{ll}
A_{2}: & L^{1}(D) \rightarrow L^{1}(D) \\
\psi(x) \rightarrow f_{i}(x) \psi(x),
\end{array}\right.\right. \\
& \begin{cases}A_{3}: & L^{1}(D) \rightarrow L^{1}(D) \\
& \psi(x) \rightarrow \int_{D} N_{\lambda}^{t}\left(x, x^{\prime}\right) \psi\left(x^{\prime}\right) d x^{\prime},\end{cases}
\end{aligned}
$$

It is clear that the operators $A_{1}, A_{2}, A_{3}$, and $A_{4}$ are bounded. In order to conclude our claim, it suffices to show that $A_{3}$ is compact on $L_{1}(D)$. In fact, by virtue of the convexity of $D, A_{3}$ is a convolution operator on $L_{1}(D)$. Since $D$ is bounded, the use of [2, Corollary 27, page 74] implies the compactness of $A_{3}$ on $L_{1}(D)$. This proves our claim.

Now the use of [7, Theorem 1] implies the continuity in the uniform topology of the map $(0, \infty) \ni t \rightarrow K U(t) K$ (because $K$ is regular) and ends the proof of the theorem. 
As a consequence of Theorem 3.2 and [7, Theorem 1] we have the following result.

COROLLARY 3.3. Let $K \in \mathscr{R}\left(L_{1}(D \times V)\right)$. Then, for $t>0$, the operators $K U(t) K$, $K V(t) K, K R_{1}(t)$ and $R_{1}(t) K$ are compact on $L_{1}(D \times V)$.

REMARK 3.3. The results of Corollary 3.3 were announced without proof in [7].

By Theorem 3.2 we conclude that if $K$ is regular, then $K \in \mathscr{P}\left(L_{1}(D \times V)\right)$. Therefore, using Theorem 2.2 and [7, Theorem 1], we obtain the following corollary.

COROLlaRY 3.4. Let $K \in \mathscr{R}\left(L_{1}(D \times V)\right)$. Then, for $t \geq 0, R_{2}(t)$ is compact on $L_{1}(D \times V)$.

REMARK 3.4. Note that the results obtained in Section 2 imply the compactness of $R_{2}(t)$ on $L_{1}(D \times V)$. This improves the second part of Theorem 3.1 in [9] where only the weak compactness of $R_{2}(t)$ on $L_{1}(D \times V)$ was obtained.

REMARK 3.5. We close this paper by observing that the class of regular collision operators was introduced in [9] and it was shown that transport equation with such collision operators have many nice properties (see [9] and [7]). We proved above that $\mathscr{P}\left(L_{p}(D \times V)\right)$ contains $\mathscr{R}\left(L_{p}(D \times V)\right)(1 \leq p<\infty)$ but we do not know yet whether these two sets are different or not.

\section{References}

[1] C. D. Aliprantis and O. Burkinshow, 'Positive compact operators on Banach lattices', Math. Z. 174 (1980), 289-298.

[2] H. Brezis, Analyse fonctionnelle: théorie et applications (Masson, Paris, 1983).

[3] R. Dautray and J. L. Lions, Analyse mathématique et calcul numérique, Tome 9 (Masson, Paris, 1988).

[4] W. Greenberg, C. Van Der Mee and V. Protopopescu, Boundary value problems in abstract kinetic theory (Birkhäuser, Basel, 1987).

[5] G. Greiner, 'Spectral properties and asymptotic behaviour of the linear transport equation', Math. Z. 185 (1984), 167-177.

[6] T. Kato, Perturbation theory for linear operators (Springer, Berlin, 1966).

[7] M. Mokhtar-Kharroubi, 'Effets régularisants en théorie neutronique', C. R. Acad. Sci. Paris, Série I 310 (1990), 545-548.

[8] — - 'Compactness properties for positive semigroups on Banach lattices and applications', Houston J. Math. 17 (1991), 25-38.

[9] —_, 'Time asymptotic behaviour and compactness in neutron transport theory', Eur. J. Mech. B Fluids 11 (1992), 39-68.

[10] A. Pazy, Semigroups of linear operators and applications to differential equations (Springer, Berlin, 1983). 
[11] P. Takac, 'Spectral mapping theorem for the exponential function in linear transport theory', Transport. Theory Statist. Phys. 14 (1985), 655-667.

[12] I. Vidav, 'Existence and uniqueness of nonnegative eigenfunction of the Boltzmann operator', $J$. Math. Anal. Appl. 22 (1968), 144-155.

[13] _ _ 'Spectra of perturbed semigroups with applications to transport theory', J. Math. Anal. Appl. 30 (1970), 264-279.

[14] J. Voigt, 'A perturbation theorem for the essential spectral radius of strongly continuous semigroups', Monatsh. Math. 90 (1980), 153-161.

[15] - 'Spectral properties of the neutron transport equation', J. Math. Anal. Appl. 106 (1985), $140-153$.

[16] L. W. Weis, 'A generalization of the Vidav-Jörgens perturbation theorem for semigroups and its application to transport theory', J. Math. Anal. Appl. 129 (1988), 6-23.

Université de Corse

Département de Mathématiques

20250 Corte

France 